\title{
Diversity of alkane hydroxylase genes on the rhizoplane of grasses planted in petroleum-contaminated soils
}

\author{
Shun Tsuboi ${ }^{12^{*}}$, Shigeki Yamamura' ${ }^{1}$, Toshiaki Nakajima-Kambe ${ }^{3}$ and Kazuhiro Iwasaki ${ }^{1}$
}

\begin{abstract}
The study investigated the diversity and genotypic features of alkane hydroxylase genes on rhizoplanes of grasses planted in artificial petroleum-contaminated soils to acquire new insights into the bacterial communities responsible for petroleum degradation in phytoremediation. Four types of grass (Cynodon dactylon, two phenotypes of Zoysia japonica, and Z. matrella) were used. The concentrations of total petroleum hydrocarbon effectively decreased in the grass-planted systems compared with the unplanted system. Among the representative alkane hydroxylase genes alkB, CYP153, almA and ladA, the first two were detected in this study, and the genotypes of both genes were apparently different among the systems studied. Their diversity was also higher on the rhizoplanes of the grasses than in unplanted oil-contaminated soils. Actinobacteria-related genes in particular were among the most diverse alkane hydroxylase genes on the rhizoplane in this study, indicating that they are one of the main contributors to degrading alkanes in oil-contaminated soils during phytoremediation. Actinobacteria-related alkB genes and CYP153 genes close to the genera Parvibaculum and Aeromicrobium were found in significant numbers on the rhizoplanes of grasses. These results suggest that the increase in diversity and genotype differences of the alkB and CYP153 genes are important factors affecting petroleum hydrocarbon-degrading ability during phytoremediation.
\end{abstract}

Keywords: Bacterial alkane hydroxylase genes, Grass roots, Petroleum contamination, Phytoremediation, Cultureindependent molecular approaches

\section{Background}

The exploration, extraction, refining, transport, and use of petroleum and derivative products has resulted in soil pollution with petroleum hydrocarbons, which is of critical environmental concern worldwide (Khan et al. 2013). Techniques for cleaning these soils include physicochemical/chemical treatments such as chemical oxidation using ferrous compounds and soil thermal desorption (Langbehn and Steinhart 1995; Ferguson et al. 2004), but these are expensive and environmentally invasive (Pandey et al. 2009; Segura et al. 2009). Biological remediation methods using plants (that is, "phytoremediation", a

\footnotetext{
*Correspondence: tsuboi.shun@nies.go.jp

${ }^{1}$ National Institute for Environmental Studies (NIES), Center for Regional Environmental Research, 16-2 Onogawa, Tsukuba 305-8506, Japan Full list of author information is available at the end of the article
}

green technology) have been recognized as excellent alternatives (Khan et al. 2004; Jain et al. 2011).

Grasses and legumes have been selected and used for phytoremediation of petroleum-polluted soils because of their tolerance to petroleum pollution. Grasses in particular are regarded as candidate plants for efficient phytoremediation because they have fibrous roots (Kaimi et al. 2007) that can loosen soil aggregates and effectively introduce oxygen, which is needed to activate alkanes by terminal oxidation by alkane hydroxylases (van Beilen et al. 2003), along root channels from the atmosphere (Adam and Duncan 1999; Merkl et al. 2005).

A primary concept of phytoremediation is that the petroleum-degrading microorganisms in the rhizosphere, which consists of rhizoplanes (the external surface of roots) and soil close to roots, have their degradation activity enhanced by exudates from the plant roots (Kuiper et al. 2001) and by molecular oxygen introduced from the

\section{㩏 Springer}


atmosphere (Adam and Duncan 1999; Merkl et al. 2005). Although previous studies reported that these plants effectively reduced petroleum concentration in the contaminated soils, presumably via stimulation of petroleumdegrading bacteria, the bacterial communities involved in the remediation remain largely unknown. Thus, characterization of the petroleum hydrocarbon-degrading bacteria on the rhizoplanes is indispensable to understanding the phytoremediation mechanisms and improving the efficiency of remediation. This study aims to acquire novel insights into the community structures and diversity of alkane-degrading bacteria on the rhizoplanes of grasses, based on culture-independent molecular approaches.

\section{Methods}

\section{Plant species}

Four types of grass were used in this study: two Japanese lawngrasses [Zoysia japonica Steud. and drought-resistant $Z$. japonica Steud. (described as "dr-Z. japonica" in this paper)], Manilagrass (Z. matrella Merr.), and Tifton Bermuda grass (Cynodon dactylon Pers.) were used in this study. The carpeting grasses were obtained from commercial gardening stores.

\section{Soil preparation, plant experiment and sampling}

To compare the diversity and phylogeny of alkanedegrading bacteria among the rhizoplane samples of the four grasses planted under the same experimental conditions, petroleum-contaminated soils $(10,000 \mathrm{mg} /$ $\mathrm{kg}$ ) were prepared by mixed commercial river sands and oil obtained from an actual petroleum-polluted site in Yamaguchi, Japan, in experimental containers (height, $500 \mathrm{~mm}$; width, $600 \mathrm{~mm}$; depth, $800 \mathrm{~mm}$; and volume, $240 \mathrm{~L}$ ). To increase the water- and nutrient-holding capacity of the soils, they were covered by a $50-\mathrm{mm}$ layer of commercial Akadama soil (small: 2-6 mm diameter, Makino, Tochigi, Japan). Sections measuring $100 \mathrm{~mm} \times 100 \mathrm{~mm}$ (length $\times$ width) were periodically cut from the $400 \mathrm{~mm} \times 600 \mathrm{~mm}$ carpeting grasses for sampling, and the roots sampled were stored at $-20{ }^{\circ} \mathrm{C}$ for molecular analysis after removing the petroleum-contaminated sands. The contaminated soils were collected to measure total petroleum hydrocarbon (TPH) concentration. Total petroleum hydrocarbon from the polluted soils was extracted and measured as soon as possible (see below). Samples collected at 856 or 891 and 494 days into the study were used to analyze alkB genes and CYP153 genes, respectively.

\section{DNA extraction from roots and detection of four alkane degradation genes (alkB, almA, CYP153 and ladA)}

DNA of root-associated bacteria was extracted from about $0.2 \mathrm{~g}$ of each root sample of the carpeting grass using the FastPrep ${ }^{\circledR}$ instrument and the FastDNA ${ }^{\circledR}$ spin kit for soil (Qbiogene, Carlsbad, CA, USA) according to the manufacturer's protocol. The PCR reaction was performed with the PCR reaction mixture containing PCR buffer with $\mathrm{MgCl}_{2}, 0.25 \mathrm{mM}$ deoxynucleotide triphosphate, $0.05 \mathrm{U}$ Ex $\mathrm{Taq}^{\circledR}$ polymerase (Takara Bio, Shiga, Japan), $2 \mu \mathrm{M}$ of each specific primer (Table 1), $2 \mu \mathrm{L}$ of template DNA and nuclease-free water to a final volume of $10 \mu \mathrm{L}$, using the Takara Thermal Cycler Dice ${ }^{\circledR}$ Gradient and Takara Thermal Cycler Dice ${ }^{\circledR}$ Touch (Takara Bio). The respective thermal conditions are shown in Additional file 1: Table S1. Successful amplification of the target genes was confirmed by electrophoresis through a $2.0 \%$ agarose gel and $0.5 \mathrm{mg} / \mathrm{L}$ ethidium bromide before a cloning procedure.

\section{Clone library constructions, sequencing and phylogenetic analysis of alkB and CYP153 genes}

Successfully amplified target genes were cloned into the pMD20-T vector with Mighty TA-cloning Kit (Takara Bio) according to the manufacturer's protocol. The constructed vectors were transformed into Escherichia coli JM109 competent cells (Takara Bio). The selected colonies were checked by direct PCR using the vector primers M13 primer M4, and M13 primer RV (Table 1) and Quick Taq ${ }^{\mathrm{TM}}$ HS DyeMix (Toyobo, Osaka, Japan) if they had an insert fragment of the correct size. From each sample, about 50 E. coli JM109 colonies with the PCR fragment of the correct size were selected randomly and used in further sequencing analysis. The positive fragments were sequenced using the BigDye ${ }^{\circledR}$ Terminator kit v.3.1 (Applied Biosystems, Carlsbad, CA, USA) and the vector primers as above, and the sequences were obtained on an Applied Biosystems 3730 DNA Analyzer (Applied Biosystems). We used BLASTx to perform a homology search of the cloned alkB and CYP153 gene sequences against the GenPept database at the National Center for Biotechnology Information (NCBI). Distance matrices were calculated based on DNADIST of PHYLIP (PHYLogeny Inference Package) 3.695 (http://evolution.genetics.washington. edu/phylip.html) and were used to group the obtained sequences into operational taxonomic units (OTUs) with a distance cut-off of 0.15 (alkB) and 0.07 (CYP153) using Mothur (Schloss et al. 2009). Rarefaction curves were calculated using " $R$ " statistics software (R Development Core Team, version 2.15.2). Evolutionary distance dendrograms were constructed by the maximum likelihood method with the Molecular Evolutionary Genetics Analysis (MEGA) 6 software package (Tamura et al. 2013). Confidence of the dendrogram topology was evaluated using bootstrap analysis with 100 resamplings. 
Table 1 PCR primers used in this study

\begin{tabular}{|c|c|c|c|}
\hline Target gene & Primer name & Sequence $\left(5-3^{\prime}\right)$ & References \\
\hline \multirow[t]{2}{*}{ alkB } & AlkB3F & TAYGGNCAYTTCTWYRTYGAGCA & Paisse et al. (2011) \\
\hline & AlkB3R & GRATTCGCRTGRTGRTC & \\
\hline \multirow[t]{2}{*}{$\operatorname{almA}$} & AlmAdf & GGNGGNACNTGGGAYCTNTT & Wang and Shao (2012) \\
\hline & AlmAdr & ATRTCNGCYTTNAGNGTCC & \\
\hline \multirow[t]{2}{*}{ CYP153 } & CYP153-F1 & ATGTTYATYGCNATGGAYCCN & Wang et al. (2011) \\
\hline & CYP153-R2 & GCGRTTVCCCATRCARCGRTG & \\
\hline \multirow[t]{2}{*}{ ladA } & ladAFR & GGCGTSTACGMCRWCTACGGYRGG & Lo Piccolo et al. (2011) \\
\hline & ladARV & GAYCTACCAGGYCGGGTCGTCG & \\
\hline \multirow[t]{2}{*}{ Vector } & M13 primer M4 & GTTTTCCCAGTCACGAC & Takara Bio \\
\hline & M13 primer RV & CAGGAAACAGCTATGAC & \\
\hline
\end{tabular}

\section{Real-time quantitative PCR (qPCR) assay}

The standard samples of the target gene quantification were constructed from retrieved and cloned DNAs from the petroleum-contaminated soil and its PCR products. The qPCR was carried out using a Takara Thermal Cycler Dice $^{\circledR}$ Real Time System Single (Takara Bio) and KOD $\mathrm{SYBR}^{\circledR}$ qPCR Mix (Toyobo) according to the manufacturers' protocols. The thermal conditions for $a l k B$ qPCR were as follows: initial denaturation at $98{ }^{\circ} \mathrm{C}$ for $2 \mathrm{~min}$, followed by 40 cycles of $98{ }^{\circ} \mathrm{C}$ for $10 \mathrm{~s}, 55^{\circ} \mathrm{C}$ for $15 \mathrm{~s}$ and $68{ }^{\circ} \mathrm{C}$ for $30 \mathrm{~s}$. For $C Y P 153$, the same thermal conditions were used with the difference that the final phase at $68^{\circ} \mathrm{C}$ was extended to $1 \mathrm{~min}$. All analyses were carried out in triplicate on each extracted DNA sample.

\section{Analytical method}

Total petroleum hydrocarbon was extracted from $0.2 \mathrm{~g}$ of sampled soil (wet weight) with $10 \mathrm{~mL}$ of polychlorotrifluoroethylene (H-997; Horiba, Kyoto, Japan) as the extraction solvent. Total petroleum hydrocarbon in the solvent was quantified with an oil-measurement instrument OCMA-350 (Horiba, Japan) based on infrared absorption analysis. A standard mixture of OCB (Horiba, Japan) consisting of 2,2,4-trimethylpentane, hexadecane and benzene was used as standard. Total petroleum hydrocarbon concentration per dry weight soil (g) was calculated and converted to $\mathrm{mg} / \mathrm{kg}$ units from the actual $\mathrm{TPH}$ concentration data and moisture ratios of soil samples.

\section{Nucleotide sequence accession numbers}

The nucleotide sequences of the partial $a l k B$ and CYP153 genes obtained in this study have been deposited into the DDBJ/EMBL/GenBank databases under the following accession numbers: LC019409 through LC019680 for the alkB genes, and LC019154 through LC019408 for the CYP153 genes.

\section{Results}

Decrease in total petroleum hydrocarbon (TPH) concentration

Total petroleum hydrocarbon concentration in the soils showed decrease in all planted systems during 856 or 891 days, respectively, while it was nearly unchanged in the unplanted system (Fig. 1). The degree of TPH concentration decrease was different among the planted systems. The $C$. dactylon-planted system in particular decreased in $\mathrm{TPH}$ concentration from approximately $7000 \mathrm{mg} / \mathrm{kg}$ to approximately $3000 \mathrm{mg} / \mathrm{kg}$.

\section{Genotypic diversity of cloned alkB and CYP153 genes}

Of the four alkane hydroxylase genes, alkB, CYP153, $\operatorname{alm} A$ and $\operatorname{lad} A$, the first two were $\operatorname{detected}$ from the rhizoplane samples. Cloned 272 alkB gene sequences were grouped into 54 OTUs. Rarefaction analysis was carried out based on these OTUs (Fig. 2a). The diversity of cloned $a l k B$ sequences was higher in the rhizoplane samples than in the unplanted oil-contaminated soil samples. Cloned 255 CYP153 gene sequences were grouped into 44 OTUs, although four of the sequences obtained were positioned outside the outgroup (Pseudomonas putida linC, accession No. AAA25810). As well as alkB genes, rarefaction analyses indicated that the genotypic diversity of cloned CYP153 genes was also higher in the rhizoplane samples than in the unplanted oil-contaminated soil samples (Fig. 2b). These observations were also supported by the diversity parameters (Additional file 1: Table S2).

\section{Phylogenetic analyses of the alkB and CYP153 genes}

alkB OTUs were phylogenetically divided into five groups (Fig. 3). Group A-I, which accounted for $23.5 \%$ of total alkB clones, was composed of Actinobacteria-related clones. Additional file 1: Figure S1a shows phylogenetic distribution in group A-I in more detail, indicating that this 


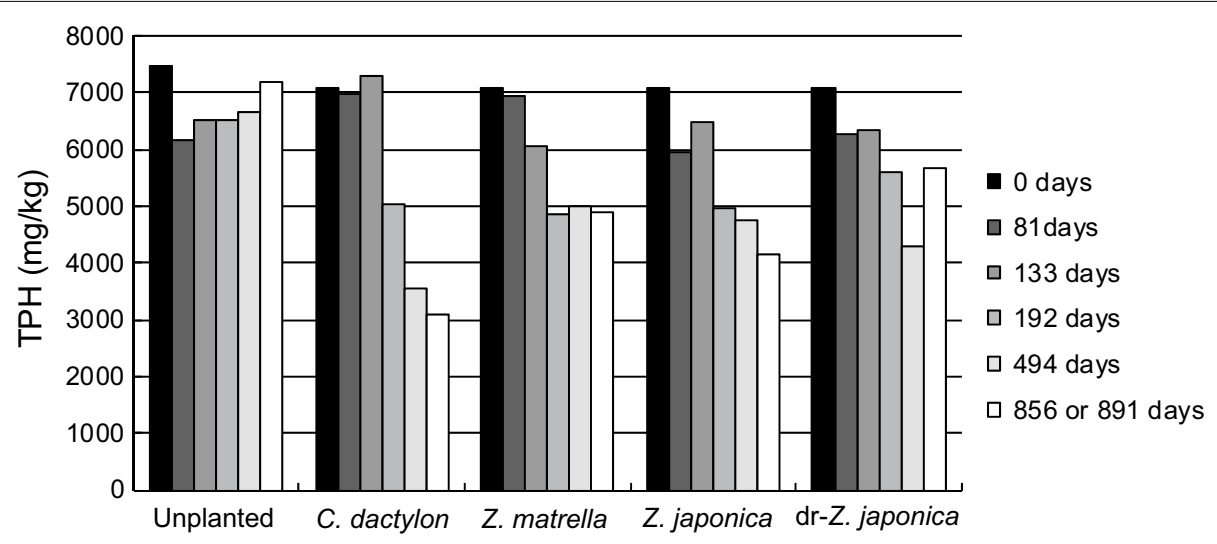

Fig. 1 Time course of total petroleum hydrocarbon (TPH) concentration in planted and unplanted systems
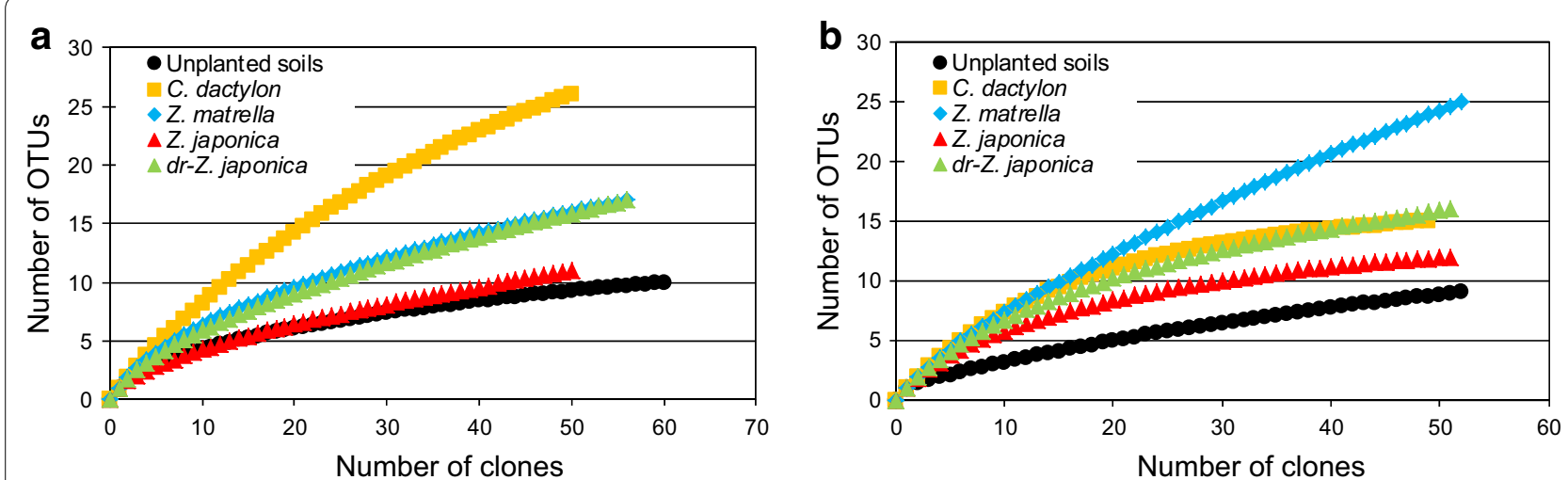

Fig. 2 Rarefaction curves of retrieved a alkB genes at 856 days (C. dactylon and Z. japonica) or 891 days (unplanted soils, Z. matrella and dr-Z. japonica) and b CYP153 genes at 494 days from four rhizoplane samples and unplanted contaminated soil samples. The $y$ axis shows the number of OTUs grouped at $85 \%$ (alkB) and $93 \%$ (CYP153) similarity

group consisted of various Actinobacterial members such as those from the genera Mycobacterium, Rhodococcus, Gordonia, Pseudonocardia, Nocardia, Marmoricola and Actinomycetospora. Genus Mycobacterium-related clones were particularly diverse. Group A-III (genus Nocardia) and A-IV (genus Mycobacterium) also consisted of Actinobacteria-related clones. Group A-V, which was the largest alkB clone group ( $44.5 \%$ of the total) in this study, was affiliated to Alpha/Gammaproteobacteria-related clones. Additional file 1: Figure S1b shows phylogenetic distribution in group A-V in more detail. The genera Stenotrophomonas and Pseudomonas of Gammaproteobacteria, and genera Caulobacter and Pseudoxanthomonas of Alphaproteobacteria, were the main members of this group. Group A-II included the alkB sequences close to those derived from Betaproteobacteria (genus Burkholderia) and Gammaproteobacteria (genera Nevskia and Solimonas) (Fig. 3). As mentioned above, Actinobacteria-related alkB clones were distributed in groups A-I, A-III and A-IV. In group
A-I, alkB genes retrieved from all systems were found (Additional file 1: Figure S1a). However, as well as group A-IV, genus Mycobacterium-related alkB clones in group A-I were found only in the rhizoplane samples. Group A-III, which included $a l k B$ genotypes derived from the genus Nocardia, was composed of only alkB clones (OTU A4) from the rhizoplane of Z. matrella (Fig. 3). Group A-V showed a prominent distribution feature (Additional file 1: Figure S1b). Genus Caulobacter-related alkB clones (OTU A1) were found in considerable numbers in all the rhizoplane samples. Meanwhile, almost all genus Pseudoxanthomonas-related alkB clones (OTU A2) were found in the unplanted oil-contaminated soil samples. Group A-II included alkB clones from all systems (Fig. 3). Genus Burkholderia-related alkB genotypes (OTU A6) were obtained from all systems. In contrast, genus Solimonas-related alkB clones (OTU A7) and genus Nevskia-related clones (OTU A3) were found in the unplanted oil-contaminated soil samples and the rhizoplane samples, respectively. 


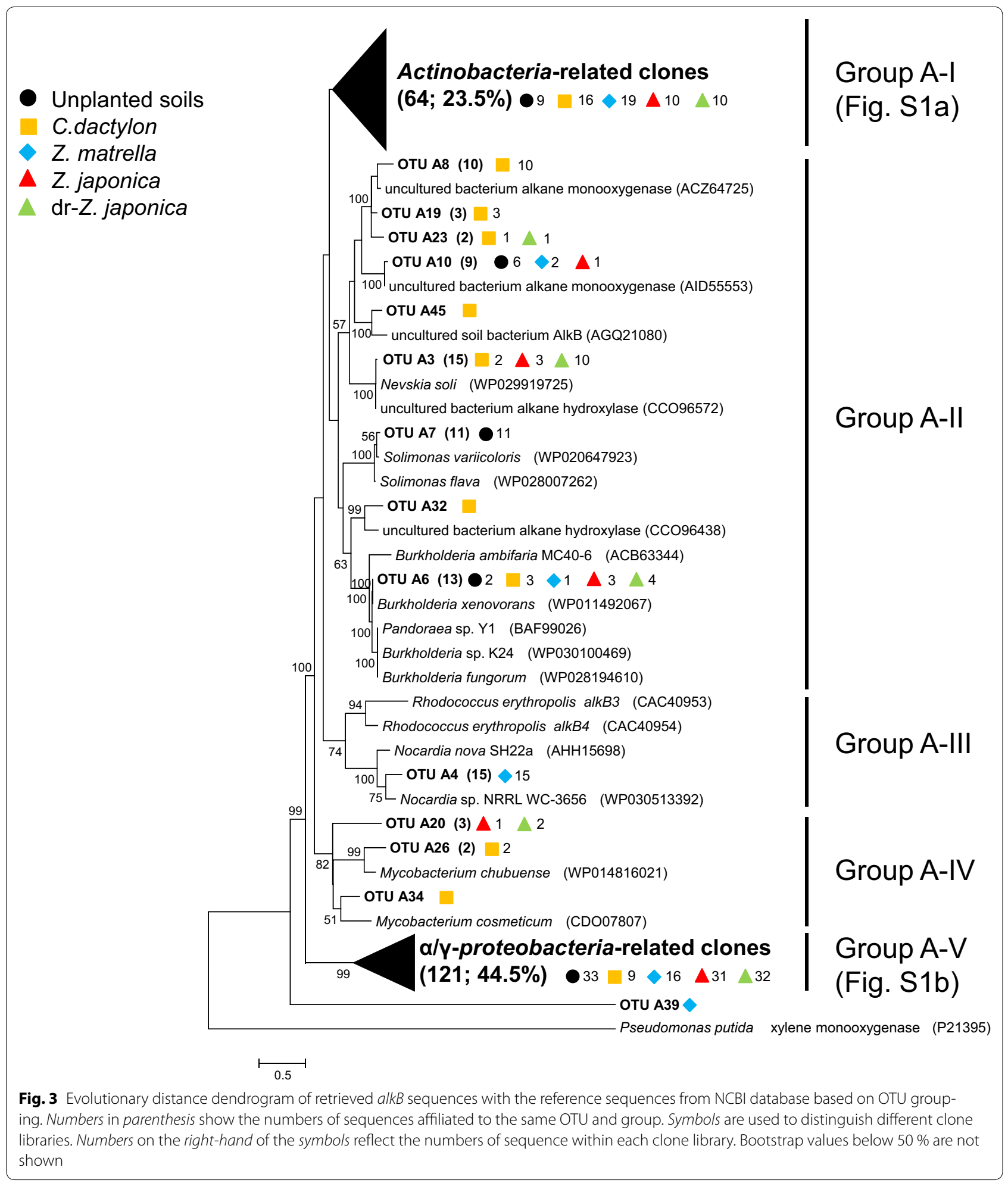

CYP153 OTUs were phylogenetically divided into five groups (Fig. 4). Group C-I, which accounted for $62.0 \%$ of total CYP153 clones, was composed of Alphaproteobacter
ia/Actinobacteria-related clones. Additional file 1: Figure S2a shows phylogenetic distribution in group C-I in more detail, indicating that this group was mainly composed of 
clones affiliated to a wide variety of Alphaproteobacteria such as the genera Bradyrhizobium, Afipia, Sphingobium, Sphingopyxis and Parvibaculum. Group C-V, the second largest CYP153 clone group (27.8\% of the total) in this study, was affiliated to Gammaproteobacteria/Actinobac teria-related clones. Additional file 1: Figure S2b shows phylogenetic distribution in group $\mathrm{C}-\mathrm{V}$ in more detail. In this group, clones closely related to genus Alcanivorax in Gammaproteobacteria and genus Aeromicrobium in Actinobacteria were mainly found. Groups C-II and C-IV were composed of Alphaproteobacteria- and Gammaproteobacteria-related clones, respectively (Fig. 4). Group C-III formed a specific branch distinct from the CYP153 gene reference sequences. Group C-I was mainly composed of CYP153 clones derived from the rhizoplane samples (Fig. 4 and Additional file 1: Figure S2a). The genus Parvibaculum-related CYP153 gene clones (OTU C2, OTU C4 and OTU C6), which were the most abundant clustered sequences in group C-I, seemed to be concentrated on the rhizoplane samples other than Z. matrella. CYP153 genes close to the uncultured Rhizobiales bacterium HF4000 48A13 (OTU C3) were also found abundantly on the rhizoplanes. Group $\mathrm{C}-\mathrm{V}$ was mainly composed of CYP153 clones derived from the unplanted oil-contaminated soil samples (Fig. 4 and Additional file 1: Figure S2b). In particular, CYP153 gene clones (OTU C1) close to an uncultured bacterium clone (accession No. BAE47472) were prominently abundant in group $\mathrm{C}-\mathrm{V}$. These clones were phylogenetically close to CYP153 genes derived from the genus Alcanivorax, but were clearly of a different genotype. The genus Aeromicrobium-related CYP153 gene clones (OTU C5 and OTU C36) that are affiliated to the phylum Actinobacteria were found only on the rhizoplane (Additional file 1: Figure S2b). Finally, groups C-II, C-III and C-IV were mainly composed of CYP153 gene genotypes from the rhizoplane of Z. matrella (Fig. 4).

\section{Sequence similarities of the alkB and CYP153 genes at amino acid levels to NCBI database}

Table 2 shows OTUs with more than $10 \%$ contribution to each of the tested systems. alkB and CYP153 sequences affiliated with OTUs in Table 2 accounted for $68.4 \%(186 / 272)$ and $65.9 \%(168 / 255)$, respectively. Genotypes (OTUs) of retrieved alkane hydroxylase genes were apparently different among all systems (Table 2). As alkB, the genotypes contained in OTU A2 and A7 were mainly found in the unplanted system, and were similar to alkB sequences of Pseudoxanthomonas spadix BD-a59 (range of similarity from 96 to $100 \%$, accession No. WP014160618) and genus Solimonas (range of similarity from 95 to $96 \%$, accession No. WP028007262 and WP020647923), respectively. OTU A1 and A5 were found in all grass-planted systems. OTU A4 (81-83\% similarity to Nocardia sp. NRRL WC-3656, accession no. WP030513392), OTU A8 (90-91 \% similarity to uncultured bacterium clone, accession no. ACZ64725) and OTU A11 (79-80 \% similarity to uncultured bacterium clone, accession no. ABB90683) were specifically detected in Z. matrella, C. dactylon and dr-Z. japonica, respectively.

As CYP153, OTU C1, which was the most similar to the uncultured bacterium clone (accession no. BAE47472), indicated a considerable large proportion $(76.9 \%$; 40/52) in the unplanted system. OTU C5 (88-90\% similarity to Aeromicrobium marinum, accession no. WP007077898) was found in the Z. matrella and dr-Z. japonica systems. OTU C4 (83-85\% similarity to the uncultured bacterium clone, accession no. CCO96723) and OTU C6 (97-100\% similarity to Parvibaculum lavamentivorans DS-1, accession no. WP012110693) were more abundant in $\mathrm{dr}-Z$. japonica and Z. japonica systems, respectively. Origins of database sequences with the highest similarity were associated with a range of environments such as oil-contaminated and uncontaminated soils, estuarine and marine sediments and seawater.

\section{Quantification of two alkane hydroxylase genes}

alkB and CYP153 genes in the rhizoplane samples were quantified by qPCR. The copy numbers (copies/g roots) of alkB and CYP153 genes ranged from $1.04 \times 10^{6}$ to $1.79 \times 10^{7}$ copies and $3.29 \times 10^{7}$ to $2.05 \times 10^{8}$ copies, respectively (Fig. 5). The abundances of both alkane hydroxylase genes did not correlate well with degradation efficiencies of TPH (Fig. 1). For instance, an effective decrease in TPH concentration was observed in the $C$. dactylon-planted system, whereas the abundance of both genes on the rhizoplane was lower than in other plants.

\section{Discussion}

Various bacterial phylogenies possess alkB and CYP153 genes, such as Alpha-, Beta-, Gamma- and Deltaproteobacteria; Actinobacteria; Bacteroides; Firmicutes; Spirochetes and Planctomycetes (Wang et al. 2010a, b; Nie et al. 2014a). In the present study, these two genes were also detected in abundance on the rhizoplanes of grasses (Fig. 5). In phylogenetic analyses of these two genes, Alpha-, Beta-, Gammaproteobacteria and Actinobacteria-related alkB and CYP153 genes were detected on the rhizoplane of grasses. These results suggest that these phylogenies play an important role in degrading the oil in the contaminated soils on the rhizoplane of grasses during phytoremediation. Culture-dependent methods show that the genera Bacillus, Ochrobactrum, Enterobacter, Pontola, Arthrobacter, Rhodococcus, Nocardia and Pseudoxanthomonas have been observed 


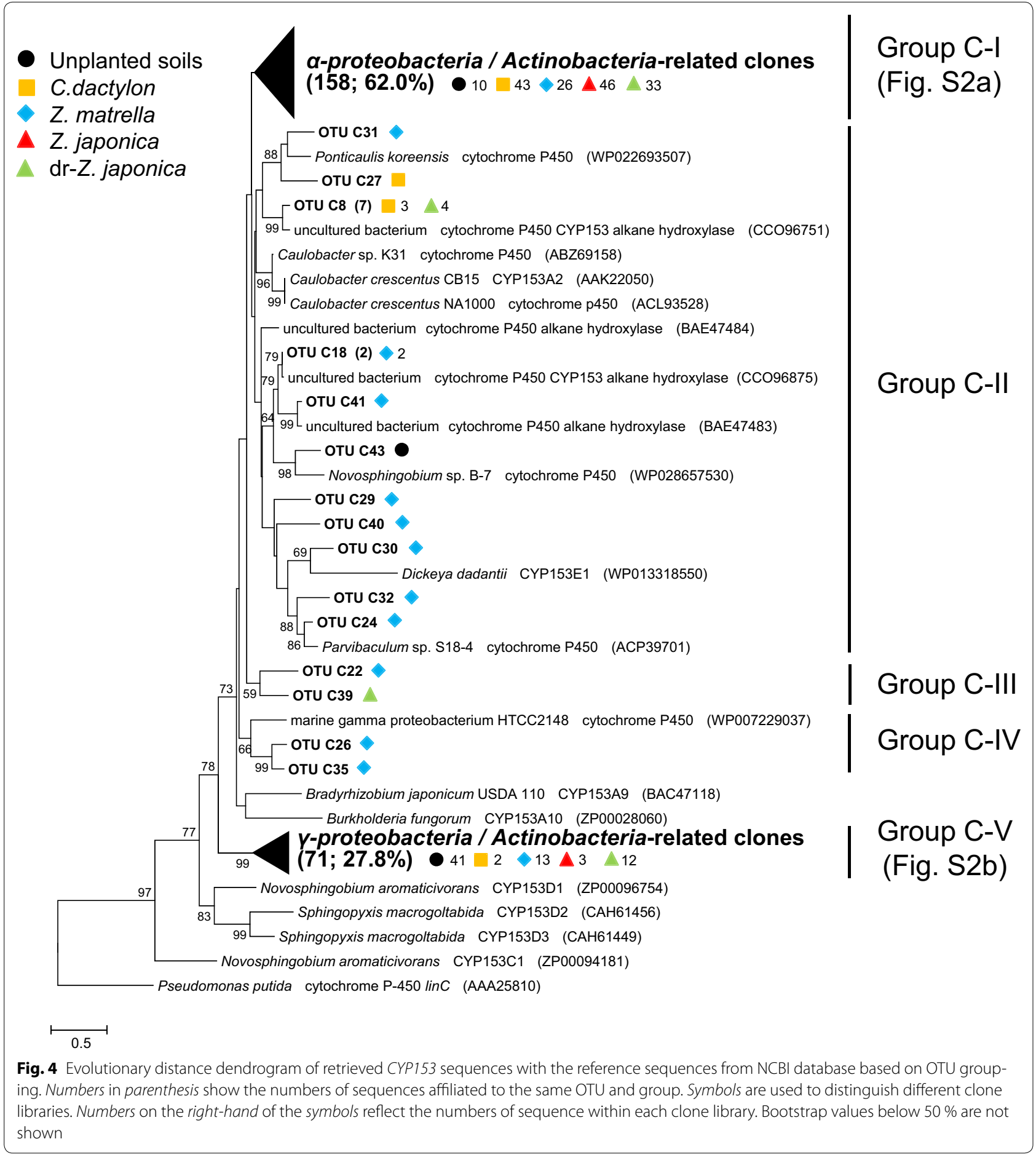

on the rhizoplanes of petroleum-contaminated soils, as the alkanes-degrading bacteria (Al-Awadhi et al. 2009). Alkane hydroxylase genes close to those of phylogenies other than those described above (such as the genera Mycobacterium, Nocardia, Aeromicrobium, Parvibaculum and Caulobacter) were also detected in abundance on the rhizoplanes in this study. Most of the retrieved sequences were also similar to clones derived from other environments such as oil-contaminated soils and estuarine sediments (Table 2). These results show that genotypes of the alkane hydroxylase genes on the rhizoplanes of grasses are more diverse than previously supposed, 
Table 2 Distribution of representative AlkB and CYP153 sequences in each system

\begin{tabular}{|c|c|c|c|c|c|}
\hline & System $(s)^{\mathbf{a}, \mathbf{b}}$ & Closest BLAST match & Range of \% ID & Sources ${ }^{c}$ & Accession no. \\
\hline \multicolumn{6}{|l|}{$\underline{a l k B}$} \\
\hline \multirow[t]{4}{*}{ OTU A1 } & $b, \underline{c}, \underline{d}, \underline{e}$ & Uncultured bacterium & $95-99$ & Oil reservoir & AGW82865 \\
\hline & & Uncultured bacterium & $94-99$ & Soil & AID55555 \\
\hline & & Uncultured soil bacterium & 99 & Pristine and hydrocarbon-contaminated soil & AGQ20909 \\
\hline & & Caulobacter sp. K31 & 94 & Chlorophenol-contaminated groundwater & YP001672212 \\
\hline OTU A2 & $\underline{a}, c$ & Pseudoxanthomonas spadix BD-a59 & $96-100$ & Gasoline-contaminated soil & WP014160618 \\
\hline \multirow[t]{3}{*}{ OTU A3 } & $b, d, \underline{e}$ & Nevskia soli & 89-99 & Soil & WP029919725 \\
\hline & & Uncultured bacterium & $89-94$ & Soil & CCO96572 \\
\hline & & Uncultured bacterium & 90 & Soil & CCO96559 \\
\hline OTU A4 & $\underline{c}$ & Nocardia sp. NRRL WC-3656 & $81-83$ & & WP030513392 \\
\hline \multirow[t]{5}{*}{ OTU A5 } & $b, c, \underline{d}, e$ & Mycobacterium tusciae & $94-96$ & Granular activated carbon & WP014814636 \\
\hline & & Mycobacterium rufum & $93-94$ & Soil & KGl67335 \\
\hline & & Mycobacterium chubuense NBB4 & 92 & Estuarine sediment & ACZ65961 \\
\hline & & Uncultured bacterium & 92 & Soil & AID23719 \\
\hline & & Uncultured bacterium & 93 & Sandy soil & ACZ64758 \\
\hline \multirow[t]{2}{*}{ OTU A7 } & $\underline{\mathrm{a}}$ & Solimonas flava & $95-96$ & Polluted farmland soil & WP028007262 \\
\hline & & Solimonas variicoloris & $95-96$ & Hexane degrading biofilter & WP020647923 \\
\hline OTU A8 & $\underline{b}$ & Uncultured bacterium & $90-91$ & Sandy soil & ACZ64725 \\
\hline OTU A9 & $\underline{c}, d$ & Uncultured bacterium & $79-97$ & Sandy soil & ACZ64717 \\
\hline OTU A10 & $\underline{a}, c, d$ & Uncultured bacterium & $96-97$ & Soil & AID55553 \\
\hline OTU A11 & $\underline{\mathrm{e}}$ & Uncultured bacterium & $79-80$ & Barley field soil & ABB90683 \\
\hline \multicolumn{6}{|l|}{$\underline{\text { CYP153 }}$} \\
\hline OTU C1 & $\underline{a}, b, d$ & Uncultured bacterium & $95-96$ & Crude oil-contaminated soil & BAE47472 \\
\hline \multirow[t]{2}{*}{ OTU C2 } & $a, \underline{b}, c, \underline{d}, \underline{e}$ & Parvibaculum lavamentivorans DS-1 & $89-100$ & Activated sludge & WP012110693 \\
\hline & & Parvibaculum lavamentivorans DS-1 & $98-99$ & Activated sludge & YP001413057 \\
\hline \multirow[t]{2}{*}{ OTU C3 } & $\mathrm{a}, \underline{\mathrm{b}}, \underline{c}, \underline{\mathrm{d}}, \mathrm{e}$ & $\begin{array}{l}\text { Uncultured Rhizobiales bacterium } \\
\text { HF4000_48A13 }\end{array}$ & $96-99$ & Coastal water & ADI19696 \\
\hline & & Uncultured bacterium & $94-97$ & Soil & CCO96903 \\
\hline OTU C4 & $a, b, c, d, \underline{e}$ & Uncultured bacterium & $83-85$ & Soil & CCO96723 \\
\hline OTU C5 & $\underline{\mathrm{c}}, \underline{\mathrm{e}}$ & Aeromicrobium marinum & $88-90$ & Sea water & WP007077898 \\
\hline \multirow[t]{2}{*}{ OTU C6 } & $b, c, \underline{d}, e$ & Uncultured bacterium & $97-100$ & Soil & CCO96726 \\
\hline & & Alpha proteobacterium MA2 & $98-99$ & Marine sediment & GAK46282 \\
\hline
\end{tabular}

a System(s) containing the respective OTUs: a, unplanted soil; b, C. dactylon; c, Z. matrella; d, Z. japonica; e, dr-Z. japonica

b System(s) with high ratios (>10\%) of each OTU were underlined

c Source of the corresponding genes from GenPept contains the genes from sole strain and the environmental clones

and the alkane-degrading rhizobacteria do not consist of rhizosphere-specific bacterial assemblages.

Both alkane hydroxylase genes show higher diversity on the rhizoplanes than in unplanted oil-contaminated soils (Fig. 2). Actinobacteria-related alkB and CYP153 genes in particular were more diverse on the rhizoplanes than in the unplanted oil-contaminated soils (Figs. 3, 4; Additional file 1: Figures S1 and S2). The Actinobacteriarelated alkane hydroxylase genes on the rhizoplanes contained the genes phylogenetically close to those of the genera Pseudonocardia, Marmoricola, Aeromicrobium, Actinomycetospora, Mycobacterium, Rhodococcus, Gordonia and Nocardia. To the best of our knowledge, this is the first study to report that alkB and CYP153, phylogenetically close to the first four of these genera, have been detected from the rhizoplanes. Whereas it has been previously discussed (Singh et al. 2007) that Actinobacteria are not seemed to be dominant in environments with continuous carbon substrate supply such as the rhizospheres of grasses, Smalla et al. (2001) found the abundance of Actinobacteria in the rhizosphere. Our results also imply that Actinobacteria were among the most diverse phyla on the rhizoplanes of grasses in oil-contaminated soils. Thus, various actinobacterial species might be some of the main contributors in degrading alkanes in contaminated soils during phytoremediation using grasses. 

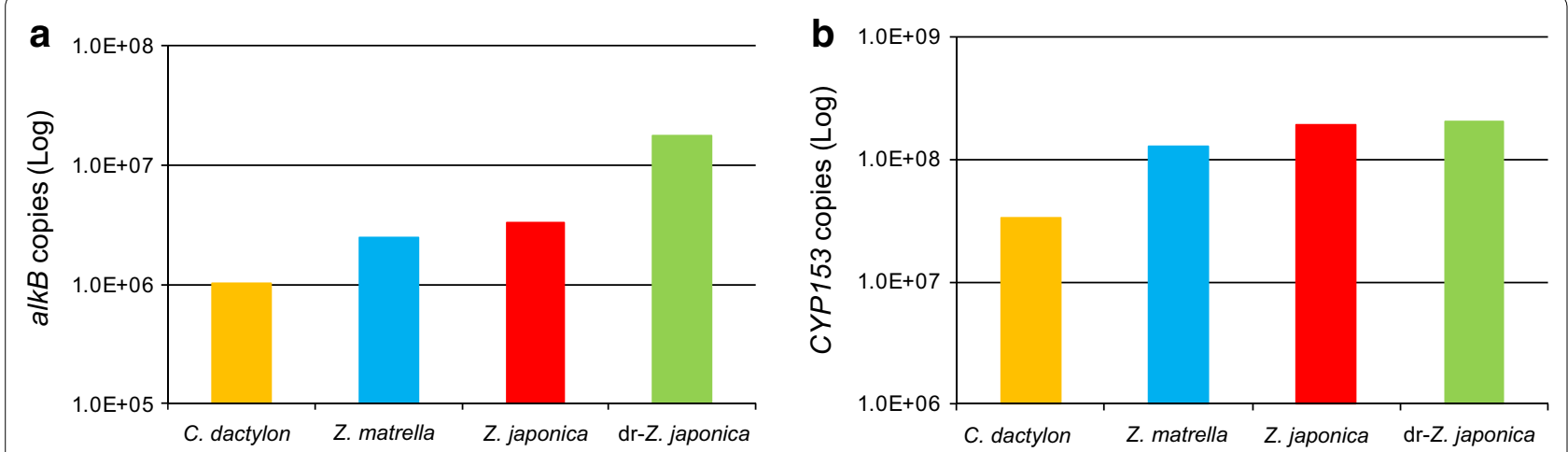

Fig. 5 Quantification of alkane hydroxylase genes on the rhizoplanes. a alkB genes at 856 days (C. dactylon and Z. japonica) or 891 days (Z. matrella and dr-Z. japonica) and $\mathbf{b}$ CYP153 genes at 494 days

The root-associated bacteria were different from bacterial communities in bulk soils (Grayston et al. 1998), although the reason(s) why diversity of hydrocarbondegrading genes on the rhizoplane increased are unclear. The genera Parvibaculum, Caulobacter and Mycobacterium, which were likely to be abundant from the detected genotypes of alkB and CYP153 genes on the rhizoplane in this study, can produce biofilms (Schleheck et al. 2000; Smit et al. 2000; Carter et al. 2003; Ojha and Hatfull 2007). Regonne et al. (2013) proposed that formation of bacterial biofilms might be associated with an increase in diversities of the polycyclic aromatic hydrocarbons (PAHs)-specific ring-hydroxylating dioxygenase alpha subunit gene responsible for phenanthrene degradation on a hydrophobic membrane laid in contaminated soils. Biofilms are considered to enhance PAH availability by increasing contact surface areas between bacteria and hydrophobic hydrocarbons (Eriksson et al. 2002). Bacterial communities in the biofilms physically and physiologically benefit each other (Stach and Burns 2002), and our results suggest that formation of biofilms is likely to help to increase genotypic diversity of alkane hydroxylase genes on the rhizoplanes.

The TPH effectively decreased in all grass-planted systems (Fig. 1), in which alkB and CYP153 genes were more diverse than in the unplanted system. However, copy numbers of both genes were not correlated with degradation efficiencies (Fig. 5). Thus, the diversity of alkane hydroxylase genes may enhance phytoremediation efficiency. It was reported that inoculation of elite alkane degraders increased degradation efficiency during phytoremediation (Soleimani et al. 2010; Afzal et al. 2011). The diversification of alkane hydroxylase genes probably increases the probability of elite alkane degraders appearing in the bacterial community on the rhizoplane. Furthermore, the co-existence of alkB and CYP153 genes in a bacterial cell enlarges the range of alkane degradation (Schneiker et al. 2006; Nie et al. 2014b). Nie et al. (2013) reported that homologues of alkane hydroxylase gene in a bacterial cell expressed at different range of alkanes. Our results suggest that the diversity and genotypes of alkane hydroxylase genes on the rhizoplane is significant in influencing alkane degradation efficiency during phytoremediation. However, further studies regarding the gene expression and activity of both alkane hydroxylases and the link between chain length of degradable alkanes and genotypic patterns of both genes is necessary to test this hypothesis.

\section{Additional file}

Additional file 1: Table S1. PCR conditions used in this study; Table S2. Diversity indices of alkB and CYP153 genes in each system; Figure S1. Evolutionary distance dendrogram of alkB (continuation of Fig. 3). (a) Group Al and (b) Group A-V. Numbers in parenthesis show the numbers of sequences affiliated to the same OTU. Symbols are used to distinguish different clone libraries. Numbers on the right-hand of the symbols reflect the numbers of sequence within each clone library. Bootstrap values below $50 \%$ are not shown; Figure S2. Evolutionary distance dendrogram of CYP153 (continuation of Fig. 4). (a) Group C-I and (b) Group C-V. Numbers in parenthesis show the numbers of sequences affiliated to the same OTU. Symbols are used to distinguish different clone libraries. Numbers on the right-hand of the symbols reflect the numbers of sequence within each clone library. Bootstrap values below 50\% are not shown.

\section{Authors' contributions}

Study conception and design: ST, TNK and KI. Acquisition of data: ST. Analysis and interpretation of data: ST and SY. Drafting of manuscript: ST and SY. All authors read and approved the final manuscript.

\section{Author details}

${ }^{1}$ National Institute for Environmental Studies (NIES), Center for Regional Environmental Research, 16-2 Onogawa, Tsukuba 305-8506, Japan. ${ }^{2}$ Present Address: National Institute for Environmental Studies (NIES), Center for Environmental Biology and Ecosystem Studies, 16-2 Onogawa, Tsukuba 305-8506, Japan. ${ }^{3}$ Faculty of Life and Environmental Sciences (Bioindustrial Sciences), University of Tsukuba, 1-1-1 Tennodai, Tsukuba 305-8572, Japan. 


\section{Acknowledgements}

The authors thank Ms. Megumi Okawa, Ms. Mahoro Tajima and Mr. Akira Tsurukoya for their assistance with collection of soils and root samples and cultivation of grasses on the oil-contaminated soils.

\section{Compliance with ethical guidelines}

\section{Competing interests}

The authors declare that they have no competing interests.

Received: 3 February 2015 Accepted: 7 September 2015 Published online: 18 September 2015

\section{References}

Adam G, Duncan HJ (1999) Effect of diesel fuel on growth of selected plant species. Environ Geochem Health 21:353-357

Afzal M, Yousaf S, Reichenauer TG, Kuffner M, Sessitsch A (2011) Soil type affects plant colonization, activity and catabolic gene expression of inoculated bacterial strains during phytoremediation of diesel. J Hazard Mater 186:1568-1575

Al-Awadhi H, El-Nemr I, Mahmoud H, Sorkhoh NA, Radwan SS (2009) Plantassociated bacteria as tools for the phytoremediation of oily nitrogenpoor soils. Int J Phytoremediat 11:11-27

Carter G, Wu M, Drummond DC, Bermudez LE (2003) Characterization of biofilm formation by clinical isolates of Mycobacterium avium. J Med Microbiol 52:747-752

Eriksson M, Dalhammar G, Mohn WW (2002) Bacterial growth and biofilm production on pyrene. FEMS Microbiol Ecol 40:21-27

Ferguson SH, Woinarski AZ, Snape I, Morris CE, Revill AT (2004) A field trial of in situ chemical oxidation to remediate long-term diesel contaminated Antarctic soil. Cold Reg Sci Technol 40:47-60

Grayston SJ, Wang S, Campbell CD, Edwards AC (1998) Selective influence of plant species on microbial diversity in the rhizosphere. Soil Biol Biochem 30:369-378

Jain PK, Gupta VK, Gaur RK, Lowry M, Jaroli DP, Chauhan UK (2011) Bioremediation of petroleum contaminated soil and water. Res J Environ Toxicol 5:1-26

Kaimi E, Mukaidani T, Tamaki M (2007) Effect of rhizodegradation in diesel-contaminated soil under different soil conditions. Plant Prod Sci 10:105-111

Khan Fl, Husain T, Hejazi R (2004) An overview and analysis of site remediation technologies. J Environ Manag 71:95-122

Khan S, Afzal M, Iqbal S, Khan QM (2013) Plant-bacteria partnerships for the remediation of hydrocarbon contaminated soils. Chemosphere 90:1317-1332

Kuiper I, Bloemberg GV, Lugtenberg BJ (2001) Selection of a plant-bacterium pair as a novel tool for rhizostimulation of polycyclic aromatic hydrocarbon-degrading bacteria. Mol Plant Microbe Interact 14:1197-1205

Langbehn A, Steinhart H (1995) Biodegradation studies of hydrocarbons in soils by analyzing metabolites formed. Chemosphere 30:855-868

Lo Piccolo L, De Pasquale C, Fodale R, Puglia AM, Quatrini P (2011) Involvement of an alkane hydroxylase system of Gordonia sp. strain SoCg in degradation of solid $n$-alkanes. Appl Environ Microbiol 77:1204-1213

Merkl N, Schultze-Kraft R, Infante C (2005) Assessment of tropical grasses and legumes for phytoremediation of petroleum-contaminated soils. Water Air Soil Pollut 165:195-209

Nie Y, Fang H, Li Y, Chi CQ, Tang YQ, Wu XL (2013) The genome of the moderate halophile Amycolicicoccus subflavus DQS3-9A $1^{\top}$ reveals four alkane hydroxylation systems and provides some clues on the genetic basis for its adaptation to a petroleum environment. PLoS One 8:e70986

Nie Y, Chi CQ, Fang H et al (2014a) Diverse alkane hydroxylase genes in microorganisms and environments. Sci Rep 4:4968. doi:10.1038/srep04968

Nie Y, Liang JL, Fang H, Tang YQ, Wu XL (2014b) Characterization of a CYP153 alkane hydroxylase gene in a Gram-positive Dietzia sp. DQ12-45-1b and its "team role" with alkW1 in alkane degradation. Appl Microbiol Biotechnol 98:163-173
Ojha A, Hatfull GF (2007) The role of iron in Mycobacterium smegmatis biofilm formation: the exochelin siderophore is essential in limiting iron conditions for biofilm formation but not for planktonic growth. Mol Microbiol 66:468-483

Paisse S, Duran R, Coulon F, Goñi-Urriza M (2011) Are alkane hydroxylase genes (alkB) relevant to assess petroleum bioremediation processes in chronically polluted coastal sediments? Appl Microbiol Biotechnol 92:835-844

Pandey J, Chauhan A, Jain RK (2009) Integrative approaches for assessing the ecological sustainability of in situ bioremediation. FEMS Microbiol Rev 33:324-375

Regonne RK, Martin F, Mbawala A, Ngassoum MB, Jouanneau Y (2013) Identification of soil bacteria able to degrade phenanthrene bound to a hydrophobic sorbent in situ. Environ Pollut 180:145-151

Schleheck D, Dong W, Denger K, Heinzle E, Cook AM (2000) An alphaproteobacterium converts linear alkylbenzene sulfonate surfactants into sulfophenylcarboxylates and linear alkyldiphenyletherdisulfonate surfactants into sulfodiphenylethercarboxylates. Appl Environ Microbiol 66:1911-1916

Schloss PD, Westcott SL, Ryabin T et al (2009) Introducing mothur: opensource, platform-independent, community-supported software for describing and comparing microbial communities. Appl Environ Microbiol 75:7537-7541

Schneiker S, Martins dos Santos VA, Bartels D et al (2006) Genome sequence of the ubiquitous hydrocarbon-degrading marine bacterium Alcanivorax borkumensis. Nat Biotechnol 24:997-1004

Segura A, Rodríguez-Conde S, Ramos C, Ramos JL (2009) Bacterial responses and interactions with plants during rhizoremediation. Microb Biotechnol 2:452-464

Singh BK, Munro S, Potts JM, Millard P (2007) Influence of grass species and soil type on rhizosphere microbial community structure in grassland soils. Appl Soil Ecol 36:147-155

Smalla K, Wieland G, Buchner A et al (2001) Bulk and rhizosphere soil bacterial communities studied by denaturing gradient gel electrophoresis: plant-dependent enrichment and seasonal shifts revealed. Appl Environ Microbiol 67:4742-4751

Smit J, Sherwood CS, Turner RF (2000) Characterization of high density monolayers of the biofilm bacterium Caulobacter crescentus: evaluating prospects for developing immobilized cell bioreactors. Can J Microbiol 46:339-349

Soleimani M, Afyuni M, Hajabbasi MA, Nourbakhsh F, Sabzalian MR, Christensen $\mathrm{JH}$ (2010) Phytoremediation of an aged petroleum contaminated soil using endophyte infected and non-infected grasses. Chemosphere 81:1084-1090

Stach JE, Burns RG (2002) Enrichment versus biofilm culture: a functional and phylogenetic comparison of polycyclic aromatic hydrocarbon-degrading microbial communities. Environ Microbiol 4:169-182

Tamura K, Stecher G, Peterson D, Filipski A, Kumar S (2013) MEGA6: molecular evolutionary genetics analysis version 6.0. Mol Biol Evol 30:2725-2729

van Beilen JB, Li Z, Duetz WA, Smits THM, Witholt B (2003) Diversity of alkane hydroxylase systems in the environment. Oil Gas Sci Technol 58:427-440

Wang W, Shao Z (2012) Diversity of flavin-binding monooxygenase genes ( $(\mathrm{I} \mathrm{mA})$ in marine bacteria capable of degradation long-chain alkanes. FEMS Microbiol Ecol 80:523-533

Wang L, Wang W, Lai Q, Shao Z (2010a) Gene diversity of CYP153A and AlkB alkane hydroxylases in oil-degrading bacteria isolated from the Atlantic Ocean. Environ Microbiol 12:1230-1242

Wang W, Wang L, Shao Z (2010b) Diversity and abundance of oil-degrading bacteria and alkane hydroxylase (alkB) genes in the subtropical seawater of Xiamen Island. Microb Ecol 60:429-439

Wang XB, Chi CQ, Nie Y, Tang YQ, Tan Y, Wu G, Wu XL (2011) Degradation of petroleum hydrocarbons (C6-C40) and crude oil by a novel Dietzia strain. Bioresour Technol 102:7755-7761 